\title{
Body fat and fat-free mass measured by bioelectric impedance spectroscopy and dual-energy $X$-ray absorptiometry in obese and non-obese adults
}

\author{
Paula Berstad ${ }^{1 *}$, Anna Randby ${ }^{2,3}$, Gunn Seim Ekeland ${ }^{3}$, Hege Ulveland ${ }^{4}$, Torbjørn Omland ${ }^{2,3}$ \\ and Kari Almendingen ${ }^{1,5,6}$ \\ ${ }^{1}$ Research Centre, Akershus University Hospital, Box 26, 1478 Lørenskog, Norway \\ ${ }^{2}$ Division of Medicine, Akershus University Hospital, Lørenskog, Norway \\ ${ }^{3}$ Faculty Division, Akershus University Hospital, University of Oslo, Lørenskog, Norway \\ ${ }^{4}$ Atlantis Medical College, Oslo, Norway \\ ${ }^{5}$ Department of Health, Nutrition and Management, Faculty of Health Sciences, Oslo and Akershus University College of \\ Applied Sciences, Norway \\ ${ }^{6}$ Institute of Chemistry, Biotechnology and Food Science, Norwegian University of Life Sciences, Ås, Norway
}

(Submitted 20 January 2011 - Final revision received 30 June 2011 - Accepted 30 June 2011 - First published online 31 August 2011)

\section{Abstract}

The aim of the present study was to compare body fat mass (FM) and fat-free mass (FFM) estimates by bioelectric impedance spectroscopy (BIS), with respective estimates by dual-energy X-ray absorptiometry (DXA), in obese and non-obese subjects. Body composition was measured in ninety-three obese and non-obese men and women by BIS device, BodyScout (Fresenius Kabi, Bad Homburg, Germany) and DXA device, Lunar iDXA (GE Healthcare, Madison, WI, USA). Mean difference between the methods was analysed by $t$ tests, and Bland-Altman plots were generated to further examine the differences between the methods. Mean difference between the estimates by DXA and BIS ( $\Delta_{\text {DXA-BIS }}$ and Bland-Altman $95 \%$ limits of agreement) were as follows: FM $4 \cdot 1(-2 \cdot 9,11 \cdot 2) \mathrm{kg}$ and $4 \cdot 5(-2 \cdot 9$, $11 \cdot 8) \%$, FFM $-4 \cdot 1(-11 \cdot 2,2 \cdot 9) \mathrm{kg}$ and $-4.5(-11.9,2 \cdot 9) \%$, indicating large inter-individual variation and statistically significant underestimation of FM and overestimation of FFM by BIS, as compared to DXA. The underestimation of FMkg (FM measured in kg) and overestimation of FFMkg (FFM measured in $\mathrm{kg}$ ) were more pronounced in men than in women, and the underestimation of FM\% (FM measured in percent) and overestimation of FFM $\%$ (FFM measured in percent) were more pronounced in normal weight $\left(\mathrm{BMI}=20 \cdot 0-24 \cdot 9 \mathrm{~kg} / \mathrm{m}^{2}\right)$ than in overweight and obese (BMI $\geq 25.0 \mathrm{~kg} / \mathrm{m}^{2}$ ) subjects. BIS may be suitable for classification of a population into groups according to FM and FFM. However, the large inter-individual variation suggests that this BIS device with the proprietary software is insufficient for estimation of single individual body FM and FFM.

Key words: Bioelectric impedance spectroscopy: Body composition: Dual-energy X-ray absorptiometry: Obesity: Validation

Measurement of body composition is frequently used in the assessment of nutritional status. Low body fat-free mass (FFM) may be a better determinant of malnutrition ${ }^{(1)}$ and mortality $^{(2,3)}$ in patients than low BMI. This is becoming more important as prevalence of obesity increases, and malnutrition in different patient groups may be hidden in obesity $^{(4,5)}$. Consequently, there is need for a simple, valid bedside body composition assessment method.

Bioelectrical impedance is a non-invasive, patient-friendly, portable and relatively inexpensive method for body composition assessment. Bioimpedance technology estimates body water compartments ${ }^{(6,7)}$ on the basis of variation in electrical conductivity between different body tissues. In bioelectric impedance spectroscopy (BIS), electric current at a spectrum of frequencies is led through the body via electrodes placed on the skin. Resistivity of extracellular water (ECW) and intracellular water (ICW) compartments against the current varies for different frequencies. High-frequency currents penetrate through cell membranes, whereas low-frequency currents pass through the body, mainly in ECW, without penetration into cells. The measured impedance spectral data are fitted into a physical model, giving estimates for ECW and total body water (TBW) volumes. Using these estimates and measured subject weight and height, ICW, and further, body

Abbreviations: BIS, bioelectric impedance spectroscopy; BMC, bone mineral content; DXA, dual-energy X-ray absorptiometry; $\Delta_{\mathrm{DXA}-\mathrm{BIS}}$, difference between the methods DXA and BIS; ECW, extracellular water; ExF, excess fluid; FFM, fat-free mass; FFMkg, fat-free mass measured in kg; FFM\%, fat-free mass measured in percent; FM, fat mass; FMkg, fat mass measured in $\mathrm{kg}$; FM\%, fat mass measured in percent; ICW, intracellular water; LTM, lean tissue mass; TBW, total body water; TW, total water.

*Corresponding author: Dr P. Berstad, fax +47 67968860, email p.m.berstad@medisin.uio.no 
FFM and fat mass (FM) can be calculated. BIS is not limited to constant body geometry and relative hydration degree of $\mathrm{FFM}^{(6,8,9)}$, which both vary in obesity ${ }^{(8,10)}$. Therefore, as compared to other bioelectrical impedance methods, BIS has higher sensitivity to predict water compartments and FFM in obese subjects or subjects with altered fluid balance ${ }^{(6,9,11)}$.

In order to use BIS for FFM estimation in clinical practice on both overweight and normal weight subjects, it has to be validated in a population with a wide spectrum of BMI. Although BIS is supposed to estimate body fluid compartments accurately in normal, healthy subjects ${ }^{(9)}$, the validity may be reduced in obesity ${ }^{(12,13)}$ and in patients in a malnourished state ${ }^{(14)}$. Dual-energy X-ray absorptiometry (DXA) is a widely used reference method for body FFM and FM measurements ${ }^{(9)}$. A recent study on cardiac patients concluded that the BIS device, BodyScout (Fresenius Kabi, Bad Homburg, Germany) only slightly overestimated FFM compared to DXA, but that the large inter-individual variation between the methods indicates that the device does not suit for body composition measurement at the individual level ${ }^{(15)}$. It remains unclear whether the validity of FFM and FM estimates by this BIS device differs between obese and non-obese subjects.

The aim of the present study was to examine the validity of BodyScout estimates of FM and FFM of the device, compared to DXA estimates, in obese and non-obese adults.

\section{Methods}

\section{Subjects}

The participants in the present study were recruited from The Akershus Sleep Apnea Project, a population-based cross-sectional study in Norway ${ }^{(16)}$. The aim of the twophase main study was to assess the prevalence of sleep apnea in Norway. A total of 30000 subjects aged 30-65 years and living in three different counties in Norway were randomly drawn from the National Population Registry and invited to respond to a questionnaire. A sub-group of 518 responders was further selected for a clinical examination.

We invited 150 randomly drawn participants for clinical examination to the present sub-study by telephone. Proportion of invited subjects with BMI $\geq 30 \cdot 0 /<30 \cdot 0 \mathrm{~kg} / \mathrm{m}^{2}$ was $80 / 20$. A total of 108 subjects gave their consent for participation, whereas forty-two refused to participate. Among those who accepted to participate, eleven subjects were unable to meet up at the measurement appointment. A total of ninetyseven subjects were included in the present study, which included anthropometric measurements, a DXA analysis and a BIS test at the Akershus University Hospital, Lørenskog, Norway. The inclusion criterion for the subjects in the clinical sub-study was not to receive treatment for obstructive sleep apnea. Subjects with significant co-morbid conditions such as cancer, heart failure or severe chronic obstructive pulmonary disease were excluded. Further exclusion criteria in the present study were pregnancy, lack of safe contraception in fertile women and those with intra-cardiac device, cochlea implant or large metallic implants in their body.
The present study was conducted according to the guidelines laid down in the Declaration of Helsinki, and all procedures were approved by the Regional Ethics Committee, The Norwegian Directorate of Health and Norwegian Social Science Data Services. Written informed consent was obtained from all subjects. The radiation dose in DXA (0.05-0.15 Gy) was considered very low, less than a standard chest X-ray.

\section{Measurement procedure}

The data were collected between May and September 2008. After fasting for a minimum of $8 \mathrm{~h}$ and after avoiding hard physical exercise for $24 \mathrm{~h}$, participants were met at the orthopaedic outpatient clinic in the morning. In all, five subjects reported not being in a fasting state. A trained health specialist (a dietitian (H. U.) or a master of science in physical activity and health (G. S. E.)) performed DXA scanning, the BIS test, and took the blood pressure and anthropometric measurements that included height, weight and waist, hip and arm circumferences. The participants also filled in a lifestyle questionnaire covering questions on physical activity, weight history, recent weight loss and chronic diseases and disabilities limiting physical activity.

\section{Anthropometric measurements}

All anthropometric measurements were conducted while the participant was without shoes and wearing only underwear. Height was measured to the nearest $\mathrm{cm}$, and body weight was measured to the nearest $0 \cdot 1 \mathrm{~kg}$ with a digital scale (Seca; Seca Deutchland, Hamburg, Germany). Mid-upper arm circumference was measured and standardised in the non-dominant arm using a scale band at the mid-point of the upper arm. Waist and hip circumferences were measured with a scale band, with the participant standing. Waist circumference was measured between the lowest ribs and crista iliaca after an out-breath. Hip circumference was measured at the level of the thickest point of nates.

\section{Dual-energy X-ray absorptiometry}

The DXA instrument used in the present study was Lunar iDXA (GE Healthcare, Madison, WI, USA). Whole-body scans were performed according to the manufacturer's instructions, and body fat, lean tissue mass (LTM) and bone mineral content (BMC) were analysed (software enCore version 11.10; GE Healthcare). The daily quality control procedure of the device was conducted automatically, and included a sixpoint calibration of three different bone-simulating chambers of known BMC and three different soft tissue-simulating chambers of known fat percentage. The program automatically determined total body measurement mode according to participant thickness (thick $>25 \mathrm{~cm}$; standard $13-25 \mathrm{~cm}$ and thin $<13 \mathrm{~cm})^{(17)}$. DXA estimate of FFM was calculated as a sum of LTM and BMC estimates. Precision of LTM and BMC estimates of the Lunar iDXA device has been found satisfactory by others (CV 0.5 and $0.6 \%$, respectively) ${ }^{(18)}$. 
A total of nineteen subjects were too large for the DXA instrument. They were scanned one-sided, and the instrument estimated measurement results for the whole body. Agreement in fat and lean mass estimates between whole-body DXA scan and estimates assessed by half-body DXA scan in obese subjects has been reported ${ }^{(19)}$.

\section{Bioelectric impedance spectroscopy}

We conducted the measurement after the participant had taken off belts, watches, jewellery and other possibly electromagnetic objects and rested in a supine position for $5 \mathrm{~min}$. During the measurement, the participant was lying in a supine position with four tactile electrodes placed on the skin, two on the dorsal surface of the dominant hand/wrist and two on the parallel foot/ankle according to the manufacturer's instructions. The BIS analyser used was BodyScout (Fresenius Kabi). The BodyScout device covers fifty frequencies in a range of $5 \mathrm{kHz}$ to $1 \mathrm{MHz}$. The proprietary software extrapolates ECW and ICW using the Hanai equation ${ }^{(20)}$, as described by Moissl et al. ${ }^{(21)}$. The BodyScout estimates of FM and FFM are not sex specific and do not expect a fixed ECW:ICW ratio. The body composition model used in the BodyScout software is based on a principle that whole-body mass $\left(M_{\mathrm{WB}}\right)$ is a sum of three compartments: normally hydrated adipose tissue mass $\left(M_{\mathrm{NH}} \_\mathrm{AT}\right)$, normally hydrated LTM $\left(M_{\mathrm{NH} \_\mathrm{LT}}\right)$ and excess fluid $(\mathrm{ExF})$ mass $\left(M_{\mathrm{ExF}}\right)$, as described by Chamney et al. ${ }^{(22,23)}$ :

$$
M_{\mathrm{WB}}=M_{\mathrm{NH} \_\mathrm{AT}}+M_{\mathrm{NH} \_\mathrm{LT}}+M_{\mathrm{ExF}}
$$

The total water (TW), ICW and ECW components of $M_{\text {NH_LT }}$ and $M_{\mathrm{NH} \_\mathrm{AT}}$ are defined by equations $2-7$.

$$
\begin{gathered}
H_{\mathrm{TW} \_\mathrm{NH} \_\mathrm{LT}}=M_{\mathrm{TW} \_\mathrm{NH} \_\mathrm{LT}} / M_{\mathrm{NH} \_\mathrm{LT}} \\
H_{\mathrm{ECW} \_\mathrm{LT}}=M_{\mathrm{ECW} \_\mathrm{NH} \_\mathrm{LT}} / M_{\mathrm{NH} \_\mathrm{LT}} \\
H_{\mathrm{ICW} \_\mathrm{NH} \_\mathrm{LT}}=M_{\mathrm{ICW} \_\mathrm{NH} \_\mathrm{LT}} / M_{\mathrm{NH} \_\mathrm{LT}} \\
H_{\mathrm{TW} \_\mathrm{NH} \_\mathrm{AT}}=M_{\mathrm{TW} \_\mathrm{NH} \_\mathrm{AT}} / M_{\mathrm{NH} \_\mathrm{AT}} \\
H_{\mathrm{ECW} \_\mathrm{NH} \_\mathrm{AT}}=M_{\mathrm{ECW} \_\mathrm{NH} \_\mathrm{AT}} / M_{\text {NH_AT }} \\
H_{\mathrm{ICW} \_\mathrm{NH} \_\mathrm{AT}}=M_{\mathrm{ICW} \text { _NH_AT }} / M_{\mathrm{NH} \_\mathrm{AT}}
\end{gathered}
$$

where $H_{\mathrm{TW} \_N H \_L T}, H_{\mathrm{ECW} \_\mathrm{LT}}$ and $H_{\mathrm{ICW} \_\mathrm{NH} \_\mathrm{LT}}$ are fractional mass of TW, ECW and ICW in normally hydrated lean tissue, and $H_{\text {TW_NH_AT }}, H_{\text {ECW_NH_AT }}$ and $H_{\text {ICW_NH_AT }}$ are fractional mass of TW, ECW and ICW in normally hydrated adipose tissue, respectively. In the BodyScout software, total body FM in $\mathrm{kg}$ (FMkg) is related to $M_{\mathrm{NH} \_\mathrm{AT}}$, as given in equation 8 .

$$
\mathrm{FMkg}=M_{\mathrm{NH} \_\mathrm{AT}} \times\left(1-H_{\mathrm{TW} \_\mathrm{NH}}{ }_{\mathrm{AT}}-K_{\mathrm{AR}}\right)
$$

where $K_{\mathrm{AR}}$ is the ratio of residual adipose components (solids, mainly protein and mineral) to $M_{\mathrm{NH} \_\mathrm{AT}}$, with a value of typically $0 \cdot 05^{(24,25)}$. FFMkg (FFM measured in $\mathrm{kg}$ ) is calculated by the software by extracting FMkg from body weight.

BIS estimate of FFM (FFMkg TBW/0.73 ) was also calculated directly from TBW, according to equation 9, based on the assumption of a $73 \%$ hydration of the $\mathrm{FFM}^{(26)}$.

$$
\text { FFMkg }_{\mathrm{TBW} / 0.73}=\mathrm{TBW} / 0.73
$$

Further, $\mathrm{FMkg}_{\mathrm{TBW} / 0.73}$ was calculated as $\mathrm{FFMkg}_{\mathrm{TBW} / 0.73}$ extracted from body weight.

\section{Lifestyle questionnaire}

In the lifestyle questionnaire, the participants were asked to report how often during the last year they were involved in non-vigorous and vigorous physical activities, choosing between four levels for both: never, $<1 \mathrm{~h} /$ week, $1-2 \mathrm{~h} /$ week and $3 \mathrm{~h} /$ week or more. They were also asked to report their habitual activity level with four choices: sedate, light activity $4 \mathrm{~h} /$ week or more, condition training $4 \mathrm{~h} /$ week or more and regular hard physical training. The participants were asked to describe their smoking status by choosing between non-smoker and current smoker, and to give the number of cigarettes smoked daily, years since start and cessation of smoking and how many years totally smoked. The participants were asked to assess their body weight at ages 18, 30, 40, 50 and 60 years, 6 and 12 months before the study date and the lowest and highest weight since the age of 18 years. The questionnaire also included a question on whether the participant had one or more chronic diseases or disabilities limiting physical activity, and if answering 'Yes', the participant was asked to write down the disease/disability.

\section{Pilot study}

We conducted a pilot study on the anthropometric, BIS and DXA measurements in twenty hospital employees and other voluntary subjects recruited by the project team in order to study variability in measurements between the two project team members who took the measurements $(H$. U. and G. S. E.). There was no statistically significant difference in the measurements of arm, waist and hip circumference or BIS and DXA measurements between the measurers (one-sample $t$ test, all $P \geq 0 \cdot 21$ ). There was a statistically significant slight difference between the measurers in height and weight measurements (mean differences $0 \cdot 1 \mathrm{~cm}$ and $0 \cdot 1 \mathrm{~kg}$ ), but this difference was without clinical significance.

\section{Statistical analysis}

Power calculation. Assuming that the participants have an average weight of $90 \mathrm{~kg}$ and $40 \%$ fat gave an estimated total FM of around 36 (SD 3) kg. Given an $\alpha$ of $5 \%$ and statistical power $(1-\beta)$ of $80 \%$, the minimum number of obese subjects to be included in each group, to detect a difference of $2 \mathrm{~kg} \mathrm{FM}$, was then $n=2 \times(3 / 2)^{2} \times 7.9=35$ per group. We also invited twenty healthy and normal weight persons 
to make sure to had a control group with optimal measurement conditions and results.

Data analysis. There is a considerable difference between the BIS and DXA technologies in estimating FMkg and FFMkg. In DXA, FM is directly estimated in $\mathrm{kg}$, and FFMkg can be calculated as the sum of LTM and BMC estimates in kg. The percentage estimates of FM and FFM are calculated using the sum of all body mass compartments in $\mathrm{kg}$ as body weight. In BIS, body weight obtained from an independent measurement (a digital scale in the present study) is inserted into the software before the measurements. FMkg and FFMkg estimates are calculated using the percentage estimates of these and the given body weight in $\mathrm{kg}$. The mean body weight measured by the digital scale in the present study was 0.5 (SD 0.8$) \mathrm{kg}$ higher than the mean DXA estimate of body weight. However, this did not significantly differ between obese and other subjects, overweight and normal weight subjects or between half- and whole-DXA-scanned subjects (data not shown). Consequently, the FMkg and FFMkg estimates of DXA were converted to values, the sum of which totalled body weight by the digital scale. We calculated physical activity score as a sum of scores from non-vigorous and vigorous activity, giving scores $0-3$ for the four choices of non-vigorous activity and double scores for the respective choices of vigorous activity. This resulted in total physical activity scores ranging from 0 to 9 . We further categorised the total physical activity scores into low $(\leq 3)$, middle (4-6) and high (7-9) activity groups. We gave score 0-2 for the habitual physical activity choices, combining the two choices with highest activity. Smoking status was reported as never, current and ex-smoker. Weight change during the preceding 12 months was calculated using the self-reported weight for 12 months earlier and the measured weight. Diseases and disabilities limiting physical activity were categorised according to type of disease/disability. Descriptive statistics are reported as distributions and as mean values with standard deviations. Bland-Altman plots ${ }^{(27)}$, a well-known tool for comparing a new and a referent method, were used to present differences between FM and FFM estimates by DXA and BIS. The limits of agreement between the two methods were defined as mean difference $\pm 1.96 \mathrm{SD}^{(27)}$. We tested mean difference between the methods $\left(\Delta_{\text {DXA-BIS }}\right)$ with paired-samples $t$ test, and used independent-samples $t$ test (if two subgroups) or one-way ANOVA (if three subgroups) to analyse differences in $\Delta_{\text {DXA-BIs }}$ between subgroups. We considered two-tailed $P<0.05$ as statistically significant. We conducted the statistical analysis using software SPSS 17.0.1 for Windows (SPSS Inc., Chicago, IL, USA).

\section{Results}

Subject characteristics are as described in Table 1. A total of ninety-three participants (61\% males) with successful measurements were included in the study, with a mean age of 51 years. The majority of the participants (fifty-nine subjects, $63 \%$ ) were obese (BMI $\geq 30 \cdot 0 \mathrm{~kg} / \mathrm{m}^{2}$ ), whereas twenty-two subjects $(24 \%)$ were overweight $\left(B M I=25 \cdot 0-29 \cdot 9 \mathrm{~kg} / \mathrm{m}^{2}\right)$ and twelve subjects (13\%) had a normal BMI (20.0$24.9 \mathrm{~kg} / \mathrm{m}^{2}$ ). Some participants showed variations in the weight measured between the clinical examination of the main study and the present study measurement date, therefore the intended proportion of subjects with BMI $\geq 30 \cdot 0 /<30 \cdot 0 \mathrm{~kg} /$ $\mathrm{m}^{2}(80 / 20)$ varied to some extent. A total of $41 \%$ of the participants reported chronic diseases or disabilities limiting physical activity.

FFMkg and FFM as a proportion (FFM\%) were significantly higher in men than in women, whereas FM as a proportion (FM\%) was significantly higher in women, estimated with both DXA and BIS (all $P<0 \cdot 001$ ). There was no significant difference in FMkg between the sexes (Table 1).

\section{Fat mass comparison}

BIS significantly underestimated mean FMkg and $\mathrm{FM} \%$ as compared to DXA (Table 2 and Fig. 1(a) and (b)). Mean differences of the estimates between the methods ( $\left.\Delta_{\text {DXA-BIS }}\right)$ and Bland-Altman $95 \%$ limits of agreements were $4 \cdot 1(-2 \cdot 9$, $11.2) \mathrm{kg}$ and $4.5(-2.9,11.8) \%$ for FMkg and FM\%, respectively (Table 2 and Fig. 1(a) and (b)). The estimates by the two methods were highly correlated (Table 2). There was a statistically significant inverse correlation between the difference and mean values of FM $\left(r_{\text {Pearson }}=-0.27\right.$ and -0.54 for FMkg and FM\%, respectively, $P<0.01$ for both; Fig. 1(a) and (b)). Underestimation of FMkg was significantly more pronounced in men than in women $\left(\Delta_{\text {DXA-BIS }}(\mathrm{SD}) 4.8(3.8)\right.$ v. $3.1(3.0) \mathrm{kg}, P=0.02$; Table 3 ). Underestimation of $\mathrm{FM} \%$ was significantly more pronounced in normal weight subjects than in overweight and obese subjects $\left(\Delta_{\mathrm{DXA}-\mathrm{BIS}}(\mathrm{SD}) 6.9(2 \cdot 7)\right.$ v. $4 \cdot 1(3 \cdot 8) \%, P=0 \cdot 005$; Table 4). The estimation difference for FM\% between the two methods was significantly inversely correlated with the FM\% estimate by DXA $\left(r_{\text {Pearson }}=-0.29\right.$, $P=0.005$; data not shown). The BIS estimates calculated as $73 \%$ hydration of FFM ( $\mathrm{FMkg}_{\mathrm{TBW} / 0.73}$ and $\left.\mathrm{FM} \%_{\mathrm{TBW} / 0.73}\right)$ were significantly higher than the respective BIS estimates by the proprietary software. Consequently, the underestimation of the FM values was significantly smaller than for the estimates given by the BIS software. However, the method differences for $\mathrm{FMkg}_{\mathrm{TBW} / 0.73}$ and $\mathrm{FM} \%_{\mathrm{TBW} / 0.73}\left(\Delta_{\mathrm{DXA}-\mathrm{BIS}(\mathrm{TBW} / 0 \cdot 73)}\right)$ were statistically significant (Table 2) and all results for $\mathrm{FM}_{\mathrm{TBW} / 0.73}$ estimates were close to identical with FM estimates by BIS software (data not shown).

There was neither a statistically significant difference in the underestimation of FMkg or FM\% by BIS between obese (BMI $\geq 30.0 \mathrm{~kg} / \mathrm{m}^{2}$ ) and other subjects nor between very obese (BMI $\geq 34.0 \mathrm{~kg} / \mathrm{m}^{2}$, eighteen subjects) and other subjects, or between subjects with half- and whole-body DXA scan (data not shown). There was no difference in the underestimation through age groups, physical activity scores or recent weight change groups (data not shown).

When grouping subjects into FMkg and FM\% quartiles by BIS and DXA estimates, 74 and $73 \%$ of the subjects, respectively, were classified into the same quartile by the two methods, whereas 26 and $25 \%$, respectively, were classified into same \pm 1 quartile (Table 5 ). 
Table 1. Characteristics of study participants

(Mean values and standard deviations, number of participants and percentages, $n$ 93)

\begin{tabular}{|c|c|c|c|c|c|c|}
\hline \multirow[b]{2}{*}{ Variable } & \multicolumn{2}{|c|}{ All (n 93) } & \multicolumn{2}{|c|}{ Men ( $n$ 57) } & \multicolumn{2}{|c|}{ Women ( $n$ 36) } \\
\hline & Mean & SD & Mean & SD & Mean & SD \\
\hline Age (years) & $51 \cdot 0$ & 11.5 & $51 \cdot 7$ & $10 \cdot 5$ & 49.7 & $13 \cdot 0$ \\
\hline Weight (kg) & 94.6 & $16 \cdot 5$ & $100 \cdot 4$ & $14 \cdot 8$ & $85 \cdot 4$ & $15 \cdot 0$ \\
\hline $\mathrm{BMI}\left(\mathrm{kg} / \mathrm{m}^{2}\right)$ & $30 \cdot 9$ & 4.5 & $31 \cdot 2$ & 4.5 & 30.5 & 4.6 \\
\hline Waist:hip ratio & 0.96 & $0 \cdot 10$ & 1.01 & 0.07 & 0.88 & 0.07 \\
\hline Arm circumference $(\mathrm{cm})$ & $33 \cdot 2$ & $3 \cdot 2$ & 33.9 & $2 \cdot 8$ & $32 \cdot 0$ & 3.4 \\
\hline \multicolumn{7}{|l|}{ DXA-derived data } \\
\hline $\mathrm{FM}(\mathrm{kg})$ & $35 \cdot 2$ & $10 \cdot 2$ & 34.2 & 9.8 & $36 \cdot 7$ & $10 \cdot 7$ \\
\hline FM (\%) & $36 \cdot 9$ & $7 \cdot 2$ & 33.5 & 5.5 & $42 \cdot 3$ & $6 \cdot 2$ \\
\hline FFM (kg) & $59 \cdot 4$ & $10 \cdot 7$ & $66 \cdot 1$ & $6 \cdot 7$ & $48 \cdot 6$ & $6 \cdot 0$ \\
\hline FFM (\%) & $63 \cdot 1$ & $7 \cdot 2$ & $66 \cdot 5$ & 5.5 & $57 \cdot 7$ & $6 \cdot 2$ \\
\hline \multicolumn{7}{|l|}{ BIS-derived data } \\
\hline ECW:ICW ratio & 0.80 & 0.08 & 0.78 & 0.07 & 0.83 & 0.08 \\
\hline FM (kg) & $31 \cdot 1$ & $11 \cdot 2$ & 29.5 & 10.9 & 33.6 & $11 \cdot 2$ \\
\hline FM (\%) & 32.5 & $9 \cdot 2$ & $28 \cdot 6$ & 7.9 & $38 \cdot 6$ & $7 \cdot 7$ \\
\hline FFM $(\mathrm{kg})$ & 63.5 & $12 \cdot 0$ & $70 \cdot 9$ & 7.9 & 51.8 & $7 \cdot 0$ \\
\hline FFM (\%) & $67 \cdot 6$ & $9 \cdot 2$ & 71.4 & 7.9 & 61.5 & $7 \cdot 7$ \\
\hline$R_{\mathrm{e}}(\Omega)$ & 549 & 77 & 511 & 51 & 611 & 72 \\
\hline$R_{\mathrm{i}}(\Omega)$ & 1122 & 255 & 992 & 161 & 1329 & 240 \\
\hline Resistence at $50 \mathrm{kHz}(\Omega)$ & 449 & 72 & 410 & 44 & 510 & 64 \\
\hline Reactance at $50 \mathrm{kHz}(\Omega)$ & $51 \cdot 2$ & $7 \cdot 3$ & $49 \cdot 6$ & $6 \cdot 6$ & $53 \cdot 7$ & $7 \cdot 8$ \\
\hline \multirow[t]{2}{*}{ Phase angle $\left({ }^{\circ}\right)$} & $6 \cdot 6$ & 0.8 & 6.9 & 0.7 & $6 \cdot 0$ & 0.7 \\
\hline & $n$ & $\%$ & $n$ & $\%$ & $n$ & $\%$ \\
\hline \multicolumn{7}{|l|}{ Abdominal obesity } \\
\hline Waist circumference $>102 \mathrm{~cm}$ (males), $>88 \mathrm{~cm}$ (females) & 65 & $69 \cdot 9$ & 39 & 68.4 & 26 & $72 \cdot 2$ \\
\hline \multicolumn{7}{|l|}{ High waist:hip ratio } \\
\hline$>1.00$ (males), $>0.85$ (females) & 57 & $61 \cdot 3$ & 35 & 61.4 & 22 & $61 \cdot 1$ \\
\hline Weight reduction $\geq 10 \%$ during the last 12 months & 6 & $6 \cdot 5$ & 3 & $5 \cdot 3$ & 3 & $8 \cdot 3$ \\
\hline Weight reduction $\geq 5 \%$ during the last 12 months & 18 & $19 \cdot 4$ & 9 & $15 \cdot 8$ & 9 & $25 \cdot 0$ \\
\hline \multicolumn{7}{|l|}{ Smoking status } \\
\hline Never smoker & 40 & $43 \cdot 0$ & 27 & $47 \cdot 4$ & 13 & $36 \cdot 1$ \\
\hline Ex-smoker & 36 & $38 \cdot 7$ & 25 & 43.9 & 11 & 30.6 \\
\hline Daily smoker & 17 & $18 \cdot 3$ & 5 & 8.8 & 12 & 33.3 \\
\hline \multicolumn{7}{|l|}{ Chronic diseases/disabilities limiting physical activity } \\
\hline Combined & 15 & $16 \cdot 1$ & 9 & $15 \cdot 8$ & 6 & $16 \cdot 7$ \\
\hline Knees/hips & 9 & $9 \cdot 7$ & 4 & 7.0 & 5 & 13.9 \\
\hline Cardiac & 6 & 6.5 & 5 & 8.8 & 1 & $2 \cdot 8$ \\
\hline Back/prolaps & 3 & $3 \cdot 2$ & 3 & $5 \cdot 3$ & - & - \\
\hline Other $^{*}$ & 5 & $5 \cdot 5$ & - & - & 5 & 13.9 \\
\hline
\end{tabular}

DXA, dual-energy X-ray absorptiometry; FM, fat mass; FFM, fat-free mass; BIS, bioelectric impedance spectroscopy; ECW, extracellular water; ICW, intracellular water; $R_{\mathrm{e}}$, resistance of extracellular volume; $R_{\mathrm{i}}$, resistance of intracellular volume.

${ }^{\star}$ Rheumatic, pulmonary or psychiatric problems, fibromyalgi or others.

Table 2. Comparison of fat mass (FM) and fat-free mass (FFM) estimates by dual-energy X-ray absorptiometry (DXA) and bioelectric impedance spectroscopy (BIS) by the proprietary software $\left(\mathrm{BIS}_{\text {software }}\right)$ and by direct calculation from total body water (TBW) by BIS

(Mean values and standard deviations, $n$ 93)

\begin{tabular}{|c|c|c|c|c|c|c|c|c|c|c|c|c|}
\hline & \multicolumn{2}{|c|}{ DXA } & \multicolumn{2}{|c|}{ BIS $_{\text {software }} \dagger$} & \multirow[t]{2}{*}{$\begin{array}{l}\text { Correlation } \\
\text { (DXA - } \\
\left.\text { BIS }_{\text {software }}\right) \\
r_{\text {Pearson }}\end{array}$} & \multicolumn{2}{|c|}{$\begin{array}{c}\text { Difference } \\
\text { between the } \\
\text { methods, } \\
\Delta_{\text {DXA-BIS(software) }}\end{array}$} & \multicolumn{2}{|c|}{ BIS $_{\text {TBW/0.73 }} \ddagger$} & \multirow[t]{2}{*}{$\begin{array}{l}\text { Correlation } \\
\text { (DXA - } \\
\text { BIS } \\
\left.r_{\text {TBW/0.73) }}\right) \\
r_{\text {earson }}\end{array}$} & \multicolumn{2}{|c|}{$\begin{array}{c}\text { Difference } \\
\text { between the } \\
\text { methods, } \\
\Delta_{\text {DXA-BIS(TBW/0. .73) }}\end{array}$} \\
\hline & Mean & $\mathrm{SD}$ & Mean & SD & & Mean & SD & Mean & $S D$ & & Mean & SD \\
\hline FM (kg) & $35 \cdot 2$ & $10 \cdot 2$ & $31 \cdot 1$ & $11 \cdot 2$ & 0.95 & $4 \cdot 1^{\star \star \star}$ & 3.6 & $31 \cdot 7$ & $10 \cdot 5$ & 0.96 & $3.5^{\star \star \star}$ & $3 \cdot 1$ \\
\hline FM \% & 36.9 & $7 \cdot 2$ & 32.5 & 9.2 & 0.92 & $4 \cdot 5^{\star \star \star}$ & $3 \cdot 8$ & $33 \cdot 2$ & $8 \cdot 3$ & 0.93 & $3 \cdot 8^{\star \star \star}$ & $3 \cdot 1$ \\
\hline FFM (kg) & $59 \cdot 4$ & $10 \cdot 7$ & 63.5 & $12 \cdot 0$ & 0.96 & $-4 \cdot 1^{\star \star \star}$ & 3.6 & 62.9 & $11 \cdot 6$ & 0.97 & $-3 \cdot 5^{\star \star \star}$ & $3 \cdot 1$ \\
\hline FFM (\%) & $63 \cdot 1$ & $7 \cdot 2$ & $67 \cdot 6$ & $9 \cdot 2$ & 0.92 & $-4 \cdot 5^{\star \star *}$ & $3 \cdot 8$ & $66 \cdot 8$ & $8 \cdot 3$ & 0.93 & $-3 \cdot 8^{\star \star \star}$ & $3 \cdot 1$ \\
\hline
\end{tabular}

${ }^{\star * \star}$ Mean values between the DXA and BIS methods were significantly different $(P<0.001)$.

† FM and FFM estimated by BIS software, as described by Chamney et al. ${ }^{(22)}$.

† FM and FFM estimated by direct calculation from TBW by BIS, expecting $73 \%$ hydration of $\mathrm{FFM}^{(26)}$. 


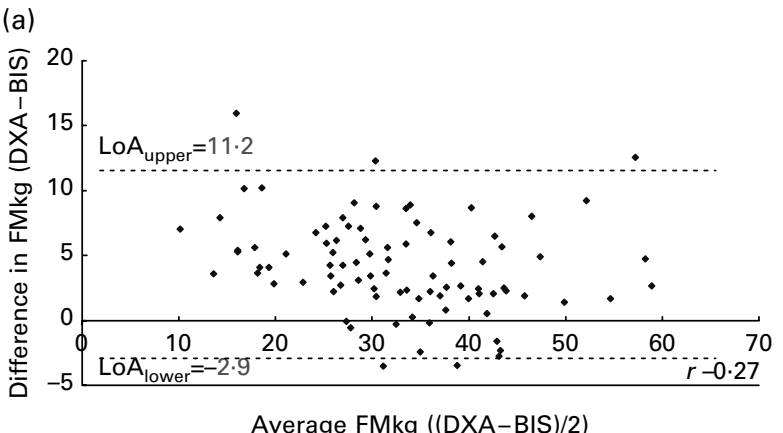

(b)

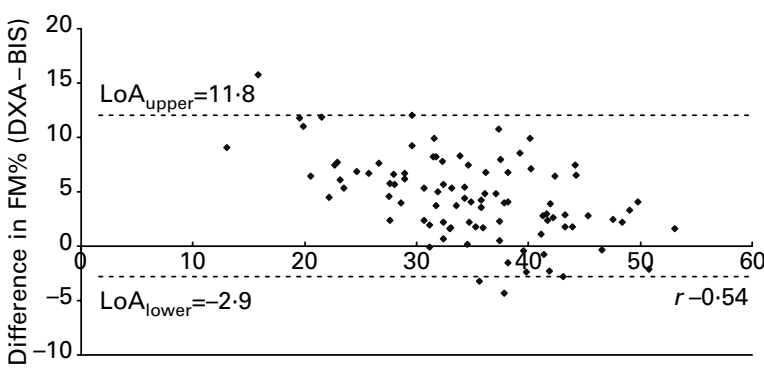

Average $\mathrm{FM} \%$ ((DXA-BIS)/2) (c)

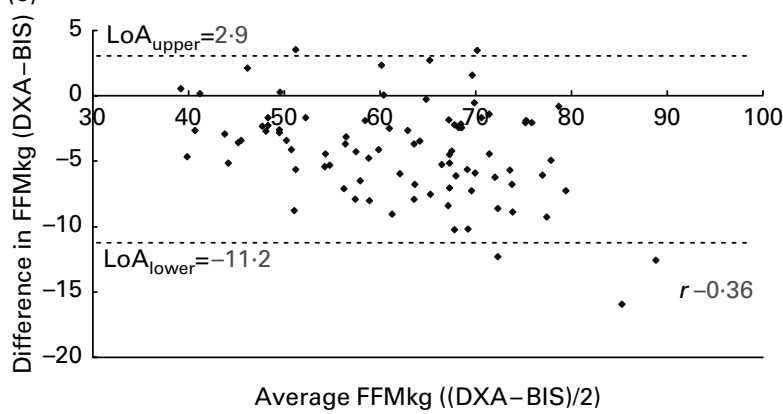

(d)

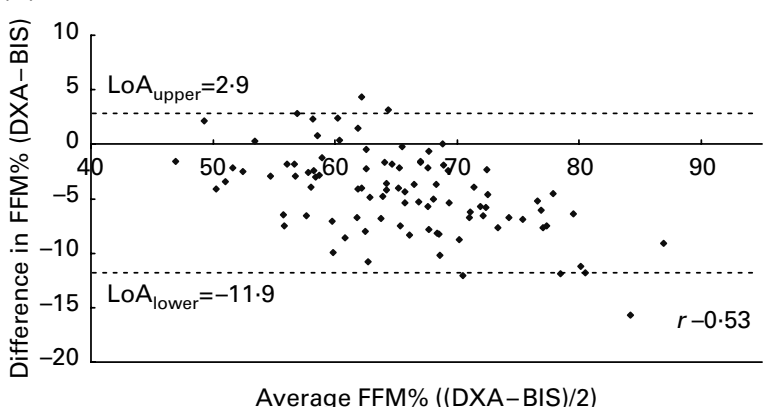

Fig. 1. Bland-Altman plots of comparisons of bioelectric impedance spectroscopy (BIS) with dual-energy X-ray absorptiometry (DXA) estimates for (a) body fat mass (FM) in kg (FMkg), (b) body FM as percentage (FM\%), (c) body fat-free mass (FFM) in kg (FFMkg) and (d) body FFM as a percentage (FFM\%) with limits of agreement (LoA) (dashed lines) and Pearson correlation coefficient $(r)(n$ 93).

\section{Fat-free mass comparison}

BIS significantly overestimated mean FFMkg and FFM\% as compared to DXA (Table 2 and Fig. 1(c) and 1(d)). $\Delta_{\text {DXA-BIS }}$ and Bland-Altman limits of agreements were $-4 \cdot 1(-11 \cdot 2$, $2.9) \mathrm{kg}$ and $-4.5(-11.9,2.9) \%$ for FFMkg and $\mathrm{FFM} \%$, respectively (Table 2 and Fig. 1(c) and (d)). The estimates by the two methods were highly correlated (Table 2). There was a statistically significant inverse correlation between the difference and mean values of FFM $\left(r_{\text {Pearson }}=-0.36\right.$ and -0.53 , for FFMkg and FFM\%, respectively, $P<0.01$ for both; Fig. 1(c) and (d)). Overestimation of the absolute FFMkg was significantly more pronounced in men than in women $\left(\Delta_{\text {DXA-BIS }}(\mathrm{SD})-4.8(3.8) v\right.$. $-3.1(3.0) \mathrm{kg}, P=0.03$; Table 3 ). Additionally, overestimation of FFM\% was significantly more pronounced in normal weight subjects than in overweight and obese subjects $\left(\Delta_{\mathrm{DXA}-\mathrm{BIS}}(\mathrm{SD})-6.9\right.$ (2.8) $v$. $-4.1(3.8) \%$, $P=0.006$; Table 4). The estimation difference for $\mathrm{FFM} \%$ between the two methods was significantly inversely correlated with the FFM $\%$ estimate by DXA $\left(r_{\text {Pearson }}=-0.33\right.$, $P=0.001$; data not shown). The BIS estimates calculated as $73 \%$ hydration of $\mathrm{FFM}$ ( $\mathrm{FFMkg}_{\mathrm{TBW} / 0.73}$ and $\mathrm{FFM} \%_{\mathrm{TBW} / 0.73}$ ) were significantly lower than the respective BIS estimates by the proprietary software. Consequently, the overestimation of the FFM values was significantly smaller than for the estimates given by the BIS software. However, the method differences for $\mathrm{FFMkg}_{\mathrm{TBW} / 0.73}$ and $\mathrm{FFM} \%{ }_{\mathrm{TBW}} / 0.73$ $\left(\Delta_{\text {DXA-BIS(TBW/0.73) }}\right)$ were statistically significant (Table 2) and all results for $\mathrm{FFM}_{\mathrm{TBW} / 0.73}$ estimates were close to identical with FFM estimates by BIS software (data not shown).
There was neither statistically significant difference in the overestimation of FFMkg or FFM\% by BIS between obese $\left(\mathrm{BMI} \geq 30 \cdot 0 \mathrm{~kg} / \mathrm{m}^{2}\right.$ ) and other subjects nor between very obese (BMI $\geq 34.0 \mathrm{~kg} / \mathrm{m}^{2}$, eighteen subjects) and other subjects, or between subjects with half- and whole-body DXA scans (data not shown). There was no difference in the overestimation through age groups, physical activity scores or recent weight change groups (data not shown).

When grouping subjects into FFMkg and FFM\% quartiles by BIS and DXA estimates, 74 and $71 \%$ of the subjects, respectively, were classified into the same quartile by the two methods, whereas 26 and 26\%, respectively, were classified into same \pm 1 quartile (Table 5 ).

\section{Discussion}

In the present validation study, we observed large interindividual variation in FM and FFM estimates between BIS

Table 3. Difference between the methods ( $\left.\Delta_{\mathrm{DXA}-\mathrm{BIS}}\right)$, where DXA is dual-energy X-ray absorptiometry and BIS is bioelectric impedance spectroscopy, in fat mass (FM) and fat-free mass (FFM) estimates in men and women

(Mean values and standard deviations)

\begin{tabular}{|c|c|c|c|c|}
\hline & \multicolumn{2}{|c|}{ Men $(n 57)$} & \multicolumn{2}{|c|}{ Women (n 36) } \\
\hline & Mean & $\mathrm{SD}$ & Mean & SD \\
\hline$\Delta \mathrm{FM}_{\mathrm{DXA}-\mathrm{BIS}}(\mathrm{kg})$ & $4 \cdot 8$ & $3 \cdot 8$ & $3 \cdot 1^{*}$ & 3.0 \\
\hline$\Delta \mathrm{FM}_{\mathrm{DXA}-\mathrm{BIS}}(\%)$ & 4.9 & 3.9 & $3 \cdot 8$ & 3.5 \\
\hline$\Delta F F M_{D X A-B I S}(k g)$ & $-4 \cdot 8$ & $3 \cdot 8$ & $-3 \cdot 1^{*}$ & $3 \cdot 0$ \\
\hline$\Delta \mathrm{FFM}_{\mathrm{DXA}-\mathrm{BIS}}(\%)$ & -4.9 & $3 \cdot 9$ & $-3 \cdot 8$ & 3.5 \\
\hline
\end{tabular}

* Mean values were significantly different from men $(P<0.05)$. 
Table 4. Difference between the methods ( $\left.\Delta_{\mathrm{DXA}-\mathrm{BIS}}\right)$, where DXA is dual-energy X-ray absorptiometry and BIS is bioelectric impedance spectroscopy, in fat mass (FM) and fat-free mass (FFM) estimates in normal weight $\left(B M I<25 \mathrm{~kg} / \mathrm{m}^{2}\right)$ and overweight $\left(B M I \geq 25 \mathrm{~kg} / \mathrm{m}^{2}\right.$ ) subjects

(Mean values and standard deviations)

\begin{tabular}{|c|c|c|c|c|}
\hline & \multicolumn{2}{|c|}{$\begin{array}{l}\text { Normal weight } \\
\text { subjects (BMl } \\
\left.<25 \mathrm{~kg} / \mathrm{m}^{2} ; n 12\right)\end{array}$} & \multicolumn{2}{|c|}{$\begin{array}{c}\text { Overweight } \\
\text { subjects }(\mathrm{BMI} \\
\left.\geq 25 \mathrm{~kg} / \mathrm{m}^{2} ; n 81\right)\end{array}$} \\
\hline & Mean & SD & Mean & SD \\
\hline$\Delta \mathrm{FM}_{\text {DXA-BIS }}(\mathrm{kg})$ & $5 \cdot 0$ & $2 \cdot 3$ & 4.0 & 3.7 \\
\hline$\Delta \mathrm{FM}_{\mathrm{DXA}-\mathrm{BIS}}(\%)$ & $6 \cdot 9$ & $2 \cdot 7$ & $4 \cdot 1^{\star \star}$ & $3 \cdot 8$ \\
\hline$\Delta F^{\prime} M_{D X A-B I S}(\mathrm{~kg})$ & $-5 \cdot 0$ & $2 \cdot 3$ & $-4 \cdot 0$ & 3.7 \\
\hline$\Delta F^{\prime} M_{\text {DXA-BIS }}(\%)$ & -6.9 & $2 \cdot 8$ & $-4 \cdot 1^{\star \star}$ & $3 \cdot 8$ \\
\hline
\end{tabular}

** Mean values were significantly different from normal weight subjects $(P<0.01)$

and DXA. We observed a significant underestimation of FM and overestimation of FFM by BIS, as compared to DXA. The underestimation of FMkg and the overestimation of FFMkg were higher in men than in women. The underestimation of $\mathrm{FM} \%$ and the overestimation of $\mathrm{FFM} \%$ were both higher at normal compared to high BMI. Furthermore, underestimation of $\mathrm{FM} \%$ was more pronounced at low FM\% and overestimation of FFM $\%$ was more pronounced at high FFM\%. The FFM and FM estimates calculated from TBW by BIS were closer to these estimates from DXA. The large inter-individual variation and heterogeneity in validity between subgroups indicate that BIS is not a suitable method for single body FM or FFM assessment at an individual level. The present BIS device, BodyScout, with the proprietary software was initially designed to estimate body liquid compartments. The present study demonstrates that it may not be equally suitable for estimation of body FM and FFM.

In the present study population with obese and non-obese subjects, using the present BIS device, the BIS estimates of FM and FFM will be significantly better if these estimates are calculated directly from TBW by BIS, expecting $73 \%$ hydration of FFM. Such observation has been done earlier by Ellegard et al. ${ }^{(14)}$. Earlier comparisons in different population groups have, in line with the present results, found large inter-individual variation in mean difference between BIS and DXA, accompanied by overestimation of $\operatorname{FFM}^{(15,28,29)}$ and under estimation of $\mathrm{FM}^{(29)}$ by BIS. Underestimation of FFM by BIS has also been reported, but in incurable cancer patients ${ }^{(14)}$. In contrast to the present study, the overestimation of FFM by BIS was, in most previous studies, in adults not related to BMI, other anthropometric variables, sex ${ }^{(28,29)}$ or magnitude of FFM and FM estimates ${ }^{(29)}$. In one study, overestimation of FFM by BIS compared to DXA was related to overweight ${ }^{(13)}$. In children, BIS has been found to overestimate FM in lean and underestimate FM in overweight subjects ${ }^{(30)}$. Higher validity of $\mathrm{FFM} \%$ and $\mathrm{FM} \%$ estimates in overweight than in normal weight subjects is, to our knowledge, reported only by the present study. The number of normal weight subjects in the present study ( $n$ 12) probably is too low to draw conclusions on this. However, the significant correlations between the method difference $\left(\Delta \mathrm{FM} \%_{\mathrm{DXA}-\mathrm{BIS}}\right.$ and $\left.\Delta \mathrm{FFM} \%_{\mathrm{DXA}-\mathrm{BIS}}\right)$ and the $\mathrm{FM} \%$ and $\mathrm{FFM} \%$ values, respectively, indicate an inverse dose-response association between validity of these estimates and adiposity of the subject.

BIS validation studies against those methods other than DXA have compared BIS estimates of ECW with dilution methods and ICW with total body potassium. These have found reduced BIS validity in disease states or obesity ${ }^{(12,21)}$. A suggested explanation for reduced validity of BIS measurements in obesity is the sensitivity of BIS to detect imbalance in body water compartments. BIS estimates of ECW and ICW are altered by changes in these, as found by Moissl in patients with renal failure and healthy subjects ${ }^{(21)}$. The ECW:ICW ratio is greater in obese subjects due to an increased amount of $\mathrm{ECW}$ and $\mathrm{TBW}^{(10)}$, as compared to non-obese subjects, leading to overestimation of FFM and underestimation of FM in obesity. Although BIS is sensitive to body water changes in normal, healthy subjects ${ }^{(9)}$, accuracy of BIS estimates of body water decreases in obesity ${ }^{(12)}$. The opposite, a larger measurement error by BIS in normal weight subjects in the present study, cannot be explained by this reduced BIS sensitivity in an abnormal body fluid balance. However, we observed larger measurement error by BIS in normal weight subjects only in the percentage proportions of FFM and FM. The reduced validity of BIS in altered fluid balance has been associated rather with the absolute estimates of ICW and ECW in litre ${ }^{(12,21)}$.

Another possible explanation for the large inter-individual variability between BIS and DXA may be a reduced validity of DXA measurements in obese subjects. Although DXA has been found to be an accurate method for body fat and lean tissue measurements ${ }^{(31)}$, underestimation of FFM in women and reduced validity in FFM estimates by truncal adiposity ${ }^{(32)}$ and increasing body size ${ }^{(33)}$ have been reported. DXA has been suspected to underestimate FM in severely obese subjects as a result of increased photon absorption ${ }^{(34)}$. If the DXA measurements in the present study were similarly biased, the observed underestimation of FM by BIS in obese subjects may have been smaller than the real underestimation in these subjects. In that case, the difference in BIS measurement error between normal weight and overweight subjects would, in reality, be smaller than observed. We did not observe a differential method difference between the subjects scanned on one side by DXA ( $n 19)$ and others. We can therefore not suspect a reduced precision of the DXA device in estimation of total body FM and FFM from the one-sided DXA scan in the present study.

Table 5. Joint classification of subjects by quartiles of fat mass (FM) and fat-free mass (FFM) estimated by dual-energy X-ray absorptiometry (DXA) and bioelectric impedance spectroscopy (BIS) ( $n$ 93)

\begin{tabular}{lccc}
\hline & $\begin{array}{c}\text { Classified } \\
\text { into same } \\
\text { quartile (\%) }\end{array}$ & $\begin{array}{c}\text { Classified } \\
\text { into same } \pm 1 \\
\text { quartile (\%) }\end{array}$ & $\begin{array}{c}\text { Classified } \\
\text { into opposite } \\
\text { quartiles (\%) }\end{array}$ \\
\hline FM (kg) & 74 & 26 & 0 \\
FM (\%) & 73 & 25 & 2 \\
FFM (kg) & 74 & 26 & 0 \\
FFM (\%) & 71 & 26 & 3 \\
\hline
\end{tabular}


It has been suggested that BIS is suitable for assessment of body composition in groups or individual body composition changes rather than determination of single individual body composition $^{(35)}$. BIS estimates of individual TBW measurements have not been considered to be valid in a population with a wide range of $\mathrm{BMI}^{(36)}$, but $\mathrm{BIS}$ has been reported to be a reproducible method within an individual ${ }^{(37,38)}$ and track intra-individual changes in TBW accurately, regardless of $\mathrm{BMI}^{(36,38)}$. Estimating changes in body composition may be the most applicable use of BIS in clinical practice, although a recent study suggested that BIS is inappropriate for this purpose also because of the large inter-individual variability $^{(15)}$. We found that BIS classifies the population relatively accurately into quartiles according to FM and FFM. This may be satisfactory for epidemiological studies. Body composition assessment is, however, mainly used in clinical studies and accurate individual measurements are necessary. Hence, correct ranging into groups is not sufficient for validity of the device.

Careful participant recruitment and standardised examination procedures are strengths of the present study. The pilot study conducted before the data collection showed agreement in measurements between the two measurers. Corrections were made in height and weight measurement procedures in order to minimise the slight measurer difference. The small number of normal weight subjects is a limitation of the present study. Use of validated regression equations has been warranted as a condition of clinical bioimpedance technology use in subjects with abnormal body shape ${ }^{(6)}$. We did not, for the purpose of the present study, develop new predicted equations based on BodyScout estimates of body fluid compartments, resistance and BMI, as the aim of the present study was to validate the BodyScout device 'as is'. The number of normal weight subjects in the present study sample would probably have been too low to develop equations useful for clinical practice.

We conclude that we found a large inter-individual agreement in FM and FFM estimates between the present BIS device and DXA. We also observed that this BIS device underestimates FM and overestimates FFM. In agreement with a recent validation study ${ }^{(15)}$, we conclude that this BIS device using the proprietary software has too low an accuracy to be used in estimation of body FM and FFM at the individual level. The absolute BIS estimates in $\mathrm{kg}$ may be less valid in men than in women, and may be less comparable with DXA estimates than the proportional estimates. In epidemiological studies, a population can be satisfactorily classified into groups by the FFM and FM estimates. Finally, we suggest that future validation studies on BIS among obese subjects include 'gold standard' methods for estimation of body fluids.

\section{Acknowledgements}

The authors are grateful to the subjects who participated in the present study and to the orthopaedic outpatient clinic at Akershus University Hospital for providing the facilities, and, in particular, to Hanna Småstu for practical help and advice. Eastern Norway Regional Health Authority RHF (no. 2006094) is acknowledged for their financial support. P. B. conducted the statistical analysis and wrote the manuscript. A. R., K. A. and T. O. designed the study. A. R. included the study subjects and coordinated data collection. G. S. E. and H. U. conducted the data collection. T. O. and K. A. obtained funding. K. A. is project leader for the ASAP study (main study) and K. A. and T. O. supervised the study. All authors critically revised the manuscript. The authors declare no conflict of interest.

\section{References}

1. Kyle UG, Morabia A, Slosman DO, et al. (2001) Contribution of body composition to nutritional assessment at hospital admission in 995 patients: a controlled population study. Br J Nutr 86, 725-731.

2. Hitzl AP, Jorres RA, Heinemann F, et al. (2010) Nutritional status in patients with chronic respiratory failure receiving home mechanical ventilation: impact on survival. Clin Nutr 29, 65-71.

3. Schols AM, Broekhuizen R, Weling-Scheepers CA, et al. (2005) Body composition and mortality in chronic obstructive pulmonary disease. Am J Clin Nutr 82, 53-59.

4. Lamb CA, Parr J, Lamb EI, et al. (2009) Adult malnutrition screening, prevalence and management in a United Kingdom hospital: cross-sectional study. Br J Nutr 102, 571-575.

5. Pressoir M, Desne S, Berchery D, et al. (2010) Prevalence, risk factors and clinical implications of malnutrition in French Comprehensive Cancer Centres. Br J Cancer 102, 966-971.

6. Kyle UG, Bosaeus I, De Lorenzo AD, et al. (2004) Bioelectrical impedance analysis-part II: utilization in clinical practice. Clin Nutr 23, 1430-1453.

7. Matthie JR (2008) Bioimpedance measurements of human body composition: critical analysis and outlook. Expert Rev Med Devices 5, 239-261.

8. Deurenberg P (1996) Limitations of the bioelectrical impedance method for the assessment of body fat in severe obesity. Am J Clin Nutr 64, 449S-452S.

9. Kyle UG, Bosaeus I, De Lorenzo AD, et al. (2004) Bioelectrical impedance analysis-part I: review of principles and methods. Clin Nutr 23, 1226-1243.

10. Waki M, Kral JG, Mazariegos M, et al. (1991) Relative expansion of extracellular fluid in obese vs. nonobese women. Am J Physiol 261, E199-E203.

11. Jaffrin MY \& Morel H (2008) Body fluid volumes measurements by impedance: a review of bioimpedance spectroscopy (BIS) and bioimpedance analysis (BIA) methods Med Eng Phys 30, 1257-1269.

12. Cox-Reijven PL \& Soeters PB (2000) Validation of bioimpedance spectroscopy: effects of degree of obesity and ways of calculating volumes from measured resistance values. Int J Obes Relat Metab Disord 24, 271-280.

13. Ward LC, Dyer JM, Byrne NM, et al. (2007) Validation of a three-frequency bioimpedance spectroscopic method for body composition analysis. Nutrition 23, 657-664.

14. Ellegard LH, Ahlen M, Korner U, et al. (2009) Bioelectric impedance spectroscopy underestimates fat-free mass compared to dual energy X-ray absorptiometry in incurable cancer patients. Eur J Clin Nutr 63, 794-801.

15. van Venrooij LM, Verberne HJ, de Vos R, et al. (2010) Preoperative and postoperative agreement in fat free mass (FFM) between bioelectrical impedance spectroscopy (BIS) and dual-energy X-ray absorptiometry (DXA) in patients undergoing cardiac surgery. Clin Nutr 29, 789-794. 
16. Hrubos-Strom H, Randby A, Namtvedt SK, et al. (2010) A Norwegian population-based study on the risk and prevalence of obstructive sleep apnea. J Sleep Res 20, 162-170.

17. GE Medical Systems (2005) Lunar iDXA Safety and Specification Manual no. L11347. Madison, WI: GE Medical Systems Lunar.

18. Hind K, Oldroyd B \& Truscott JG (2011) In vivo precision of the GE Lunar iDXA densitometer for the measurement of total body composition and fat distribution in adults. Eur J Clin Nutr 65, 140-142.

19. Rothney MP, Brychta RJ, Schaefer EV, et al. (2009) Body composition measured by dual-energy X-ray absorptiometry half-body scans in obese adults. Obesity (Silver Spring) 17, 1281-1286.

20. Hanai T (1968) Electrical properties of emulsions. In Emulsion Science, pp. 354-477. London: Academic Press.

21. Moissl UM, Wabel P, Chamney PW, et al. (2006) Body fluid volume determination via body composition spectroscopy in health and disease. Physiol Meas 27, 921-933.

22. Chamney PW, Wabel P, Moissl UM, et al. (2007) A wholebody model to distinguish excess fluid from the hydration of major body tissues. Am J Clin Nutr 85, 80-89.

23. Wabel P, Chamney P, Moissl U, et al. (2009) Importance of whole-body bioimpedance spectroscopy for the management of fluid balance. Blood Purif 27, 75-80.

24. Holliday MA (1986) Body composition and energy need during growth. In Human growth, pp. 101-117. New York, NY: Plenum Press.

25. Martin AD, Daniel MZ, Drinkwater DT, et al. (1994) Adipose tissue density, estimated adipose lipid fraction and whole body adiposity in male cadavers. Int J Obes Relat Metab Disord 18, 79-83.

26. Ellis KJ (2000) Human body composition: in vivo methods. Physiol Rev 80, 649-680.

27. Bland JM \& Altman DG (1999) Measuring agreement in method comparison studies. Stat Methods Med Res 8, $135-160$

28. Carlsson E, Bosaeus I \& Nordgren S (2004) Body composition in patients with short bowel syndrome: an assessment by bioelectric impedance spectroscopy (BIS) and dualenergy absorptiometry (DXA). Eur J Clin Nutr 58, 853-859.
29. Svantesson U, Zander M, Klingberg S, et al. (2008) Body composition in male elite athletes, comparison of bioelectrical impedance spectroscopy with dual energy X-ray absorptiometry. J Negat Results Biomed 7, 1

30. Fors H, Gelander L, Bjarnason R, et al. (2002) Body composition, as assessed by bioelectrical impedance spectroscopy and dual-energy X-ray absorptiometry, in a healthy paediatric population. Acta Paediatr 91, 755-760.

31. Svendsen OL, Haarbo J, Hassager C, et al. (1993) Accuracy of measurements of body composition by dual-energy X-ray absorptiometry in vivo. Am J Clin Nutr 57, 605-608.

32. Hansen RD, Raja C, Aslani A, et al. (1999) Determination of skeletal muscle and fat-free mass by nuclear and dual-energy X-ray absorptiometry methods in men and women aged 51-84 y (1-3). Am J Clin Nutr 70, 228-233.

33. Williams JE, Wells JC, Wilson CM, et al. (2006) Evaluation of Lunar Prodigy dual-energy X-ray absorptiometry for assessing body composition in healthy persons and patients by comparison with the criterion 4-component model. Am J Clin Nutr 83, 1047-1054.

34. Levitt DG, Beckman LM, Mager JR, et al. (2010) Comparison of DXA and water measurements of body fat following gastric bypass surgery and a physiological model of body water, fat, and muscle composition. J Appl Physiol 109, 786-795.

35. Buchholz AC, Bartok C \& Schoeller DA (2004) The validity of bioelectrical impedance models in clinical populations. Nutr Clin Pract 19, 433-446.

36. Moon JR, Tobkin SE, Roberts MD, et al. (2008) Total body water estimations in healthy men and women using bioimpedance spectroscopy: a deuterium oxide comparison. Nutr Metab (Lond) 5, 7 .

37. Andersen TB, Jodal L, Arveschoug A, et al. (2011) Precision and within- and between-day variation of bioimpedance parameters in children aged 2-14 years. Clin Nutr 30, 326-331.

38. Moon JR, Stout JR, Smith AE, et al. (2010) Reproducibility and validity of bioimpedance spectroscopy for tracking changes in total body water: implications for repeated measurements. Br J Nutr 104, 1384-1394. 